\title{
On Confluence of Infinitary Combinatory Reduction Systems
}

\author{
Jeroen Ketema ${ }^{1}$ and Jakob Grue Simonsen ${ }^{2}$ \\ 1 Department of Computer Science, Vrije Universiteit Amsterdam \\ De Boelelaan 1081a, 1081 HV Amsterdam, The Netherlands \\ jketema@cs.vu.nl \\ 2 Department of Computer Science, University of Copenhagen (DIKU) \\ Universitetsparken 1, DK-2100 Copenhagen Ø, Denmark \\ simonsen@diku.dk
}

\begin{abstract}
We prove that fully-extended, orthogonal infinitary combinatory reduction systems with finite right-hand sides are confluent modulo identification of hypercollapsing subterms. This provides the first general confluence result for infinitary higher-order rewriting.
\end{abstract}

\section{Introduction}

Lazy declarative programming employs several approaches that are well-suited for description by term rewriting. This is of interest when studying basic constructs such as lazy lists:

$$
\operatorname{from}(x, y) \leftarrow x^{\prime} \text { is } x+1, \operatorname{from}\left(x^{\prime}, z\right), y=[x \mid z]
$$

and (lazy) narrowing or residuation, in conjunction with, say, higher-order functions, e.g. the map functional:

$$
\begin{aligned}
& \operatorname{map}(f,[])=[] \\
& \operatorname{map}(f,[x \mid x s])=[f(x) \mid \operatorname{map}(f, x s)]
\end{aligned}
$$

Such a combination occurs in several pure functional languages, as well as in functional logic languages such as CurRY [1,2] and ToY [3].

An extension of term rewriting intended to model lazy computations is infinitary rewriting, a formalism allowing for terms and reductions to be infinite [4-6]. Technical properties known as strong convergence and compression furnish the computational intuition for such systems: The limit term of every infinitely long sequence of computations is also the limit of a sequence of finite computations. Unfortunately, many desirable properties of ordinary (first-order) term rewriting systems fail to hold when considering infinitary term rewriting systems (iTRSs). Furthermore, substantial care and ingenuity is needed to treat bound variables and applications in the infinitary setting, a fact already evident in infinitary lambda calculus $(\mathrm{i} \lambda \mathrm{c})[6,7]$.

While many language features require some sort of extension or restriction on the rewrite relation to model actual computations correctly (e.g. conditional 
rewriting for logic programming $[8,9]$ ), any systematic treatment of such variants of infinitary rewriting must wait until the basic theory for infinitary higher-order rewriting has been pinned down. The contribution of this paper is to do exactly that by proving a general confluence (or Church-Rosser) theorem for infinitary higher-order rewriting. Our proof follows the general outline of confluence proofs for more restricted kinds of infinitary rewriting [6], but the crucial methods we employ are adapted from van Oostrom's treatment [16] of a method by Sekar and Ramakrishnan [15]. We work with infinitary combinatory reduction systems (iCRSs), as introduced in [10].

The outline of the paper is as follows: Section 2 introduces the basic concepts, Section 3 treats developments of sets of redexes, Section 4 concerns a special class of troublesome terms: the hypercollapsing ones, and the proof methods needed to tackle them, while Section 5 provides a proof of the main result.

\section{Preliminaries}

This section briefly recapitulates basic facts concerning both ordinary and infinitary CRSs; the reader is referred to [11] for an account of CRSs, and to [10] for iCRSs.

Throughout the paper we assume a signature $\Sigma$, each element of which has finite arity. We also assume a countably infinite set of variables, and, for each finite arity, a countably infinite set of meta-variables. Countably infinite sets are sufficient, given that we can employ 'Hilbert hotel'-style renaming. We denote the first infinite ordinal by $\omega$, and arbitrary ordinals by $\alpha, \beta, \gamma, \ldots$. We use $\mathbb{N}$ to denote the set of natural numbers, starting at zero.

The standard way of defining infinite terms in infinitary rewriting is by defining a metric on the set of finite terms and letting the set of infinite terms be the completion of the metric space of finite terms $[5,7,12]$, an approach also used in [10]; here, we give a shorter, but equivalent, definition using so-called "candidate" meta-terms:

Definition 2.1. The set of (infinite) candidate meta-terms is defined by interpreting the following rules coinductively:

1. each variable $x$ is a candidate meta-term,

2. $[x] s$ is a candidate meta-term, if $x$ is a variable and $s$ is a candidate metaterm,

3. $Z\left(s_{1}, \ldots, s_{n}\right)$ is a candidate meta-term, if $Z$ is a meta-variable of arity $n$ and $s_{1}, \ldots, s_{n}$ are candidate meta-terms, and

4. $f\left(s_{1}, \ldots, s_{n}\right)$ is a candidate meta-term, if $f \in \Sigma$ has arity $n$ and $s_{1}, \ldots, s_{n}$ are candidate meta-terms.

A candidate meta-term of the form $[x] s$ is called an abstraction. Each occurrence of the variable $x$ in $s$ is bound in $[x] s$.

The set of finite meta-terms, a subset of the candidate meta-terms, is the set inductively defined by the above rules. 
Thus, $[x] x,[x] f(Z(x)), Z(Z(Z(\ldots)))$, and $Z\left([x] Z^{\prime}\left([y]\left(Z\left([x] Z^{\prime}\right) \ldots\right)\right)\right)$ are all candidate meta-terms. Moreover, $[x] x$ and $[x] f(Z(x))$ are also finite meta-terms.

As usual in rewriting, we define the set of positions of candidate meta-terms as a set of finite strings over $\mathbb{N}$, with $\epsilon$ the empty string, such that each string corresponds to the "location" of subterm. For instance, the position of $y$ in $[x] f(x, y)$ is 01 (' 0 ' to get to $f(x, y)$ and ' 1 ' to get to the second argument of $f)$. The set of positions of term $s$ is denoted $\operatorname{Pos}(s)$. If $p \in \mathcal{P} o s(s)$, then we denote by $\left.s\right|_{p}$ the subterm of $s$ at $p$ (e.g. $\left.\left.[x] f(x, y)\right|_{01}=y\right)$. The length of a position $p$ is denoted $|p|$. There is a natural well-founded (but not necessarily total) order $<$ on positions such that $p<q$ iff $p$ is a proper prefix of $q$. If $p$ and $q$ are incomparable in this order, we write $p \| q$ and say that $p$ and $q$ are parallel.

A (one-hole) context is a candidate meta-term over $\Sigma \cup\{\square\}$ where $\square$ is a fresh constant that occurs at most once in the term.

We next define the set of meta-terms:

Definition 2.2. Let $s$ be a candidate meta-term. A chain in $s$ is a sequence of (context,position)-pairs $\left(C_{i}[\square], p_{i}\right)_{i<n}$ where $n \in \omega+1$, such that for each $\left(C_{i}[\square], p_{i}\right)$ there exists a term $t_{i}$ with the property that $C_{i}\left[t_{i}\right]=\left.s\right|_{p_{i}}$ and $p_{i+1}=$ $p_{i} \cdot q$ where $q$ is the position of the hole in $C_{i}[\square]$.

$A$ chain of meta-variables is a chain $\left(C_{i}[\square], p_{i}\right)_{i<n}$ such that for each $i<n$ it holds that $C_{i}[\square]=Z\left(t_{1}, \ldots, t_{m}\right)$ with $t_{j}=\square$ for at most one $1 \leq j \leq m$.

$A$ meta-term is a candidate metaterm s such that no infinite chain of metavariables occurs in $s$.

Observe that $\square$ occurs only in $C_{i}[\square]$ if $i+1<n$, otherwise $C_{i}[\square]=\left.s\right|_{p_{i}}$. Moreover, note that candidate meta-terms such as $Z(Z(Z(\cdots Z(\cdots))))$ are not metaterms. These terms are rejected as meta-terms as the result of applying substitutions to them is generally not well-defined [10]. Note too that $\left[x_{1}\right] Z_{1}\left(\left[x_{2}\right] Z_{2}(\ldots)\right)$ is a meta-term.

We can now define terms:

Definition 2.3. A term is a meta-term without meta-variables.

As usual, we consider terms modulo $\alpha$-equivalence. Note that the definition of meta-terms only restricts meta-terms containing meta-variables, not meta-terms without meta-variables, i.e. not terms. Substitutions are defined by interpreting the ordinary rules of substitution coinductively, minding $\alpha$-conversion when applicable. We write $s[\boldsymbol{x}:=\boldsymbol{t}]$ for the substitution of a vector $\boldsymbol{t}$ of terms for a vector $\boldsymbol{x}$ of variables (of the same length) in a term $s$. An $n$-ary substitute is a mapping denoted $\underline{\lambda} x_{1}, \ldots, x_{n} . s$ or $\underline{\lambda} \boldsymbol{x} . s$, with $s$ a term, such that:

$$
(\underline{\lambda} \boldsymbol{x} . s)\left(t_{1}, \ldots, t_{n}\right)=s[\boldsymbol{x}:=\boldsymbol{t}] .
$$

A valuation $\bar{\sigma}$ is an extension of a function $\sigma$ which assigns $n$-ary substitutes to $n$-ary meta-variables. The extension maps meta-terms to terms. For instance, if $\sigma(Z)=[x] f(x)$, we have $\bar{\sigma}(g(Z, x))=g([x] f(x), x)$. As above, it is defined by interpreting the usual rules for valuations [11] coinductively.

The following is proved in [10]: 
Proposition 2.4. Let $s$ be a meta-term and $\bar{\sigma}$ a valuation. There exists a unique term that is the result of applying $\bar{\sigma}$ to $s$.

\subsection{Infinitary Rewriting}

Definition 2.5. A finite meta-term is a pattern if each of its meta-variables has distinct bound variables as its arguments. Moreover, a meta-term is closed if all its variables occur bound.

Definition 2.6. A rewrite rule is a pair $(l, r)$, denoted $l \rightarrow r$, where $l$ is a finite meta-term and $r$ is a meta-term, such that:

1. $l$ is a pattern and of the form $f\left(s_{1}, \ldots, s_{n}\right)$ with $f \in \Sigma$ of arity $n$,

2. all meta-variables that occur in $r$ also occur in $l$, and

3. $l$ and $r$ are closed.

An infinitary combinatory reduction system (iCRS) is a pair $\mathcal{C}=(\Sigma, R)$ with $\Sigma$ a signature and $R$ a set of rewrite rules.

Definition 2.7. A rewrite rule $l \rightarrow r$ is left-linear, respectively collapsing, if each meta-variable occurs at most once in $l$, respectively if $r$ has a meta-variable as root symbol. An iCRS is left-linear if all its rewrite rules are left-linear.

Definition 2.8. A pattern is fully-extended [13, 14], if, for each of its metavariables $Z$, and each abstraction $[x]$ having $Z$ in its scope, $x$ is an argument of $Z$. An iCRS is fully-extended if the left-hand sides of all rewrite rules are.

We now define redexes and rewrite steps.

Definition 2.9. Let $l \rightarrow r$ be a rewrite rule. Given a valuation $\bar{\sigma}$, the term $\bar{\sigma}(l)$ is called a $l \rightarrow r$-redex. If $s=C[\bar{\sigma}(l)]$ for some context $C[\square]$ with $\bar{\sigma}(l)$ a $l \rightarrow r$ redex and $p$ the position of the hole in $C[\square]$, then an $l \rightarrow r$-redex, or simply a redex, occurs at position $p$ and depth $|p|$ in s. Moreover, a position $q$ occurs in the redex pattern, if $q \geq p$ and if there does not exist $q^{\prime}$ such that $q \geq p \cdot q^{\prime}$ and $q^{\prime}$ is the position of a meta-variable in $l$.

$A$ rewrite step is a pair $(s, t)$, denoted $s \rightarrow t$, such that an $l \rightarrow r$-redex occurs in $s=C[\bar{\sigma}(l)]$ and such that $t=C[\bar{\sigma}(r)]$. A redex or rewrite step is collapsing if the employed rewrite rule is collapsing. It is root-collapsing if it is collapsing and if the redex occurs at position $\epsilon$.

Throughout the paper, sets of redexes are denoted by calligraphic capitals such as $\mathcal{U}$. We can now define what a transfinite reduction sequence is. The definition copies the definition from iTRSs and $\mathrm{i} \lambda \mathrm{c}$ verbatim $[5,7]$ :

Definition 2.10. A transfinite reduction sequence of ordinal length $\alpha$ is a sequence of terms $\left(s_{\beta}\right)_{\beta<\alpha+1}$ such that $s_{\beta} \rightarrow s_{\beta+1}$ for all $\beta<\alpha$. For each rewrite step $s_{\beta} \rightarrow s_{\beta+1}$, let $d_{\beta}$ denote the depth of the contracted redex. The reduction sequence is weakly convergent or Cauchy convergent if for every ordinal $\gamma \leq \alpha$ the distance between $t_{\beta}$ and $t_{\gamma}$ tends to 0 as $\beta$ approaches $\gamma$ from below. The reduction sequence is strongly convergent if it is weakly convergent and if $d_{\beta}$ tends to infinity as $\beta$ approaches $\gamma$ from below. 
Notation 2.11. By $s \rightarrow^{\alpha} t$, respectively $s \rightarrow \leq \alpha t$, we denote a strongly convergent transfinite reduction sequence of ordinal length $\alpha$, respectively of ordinal length less than or equal to $\alpha$. By $s \rightarrow t$ we denote a strongly convergent transfinite reduction sequence of arbitrary ordinal length and by $s \rightarrow^{*} t$ we denote a reduction sequence of finite length. Reduction sequences are usually ranged over by capitals such as $D, S$, and $T$. The concatenation of two reduction sequences $S$ and $T$ is denoted by $S ; T$. Note that the concatenation of any finite number of strongly convergent reductions is a strongly convergent reduction.

Lemma 2.12 (See [10]). If $s \rightarrow t$, then the number of steps contracting redexes at depths less than $d \in \mathbb{N}$ is finite for any $d$.

As in [5-7], we consider strongly converging reduction sequences. This ensures that we can restrict our attention to reduction sequences of length at most $\omega$ by the so-called compression property:

Theorem 2.13 (Compression, see [10]). For every fully-extended, left-linear iCRS, if $s \rightarrow^{\alpha} t$, then $s \rightarrow \leq \omega t$.

Left-linearity and fully-extendedness ensure no redex is created by either making two subterms equal or erasing some variable in an infinite number of steps. As shown in [10], they cannot be omitted from the theorem. In the remainder we work exclusively with orthogonal systems; these are defined as in the finite case:

Definition 2.14. Let $R=\left\{l_{i} \rightarrow r_{i} \mid i \in I\right\}$ be a set of rewrite rules.

1. $R$ is non-overlapping if it holds that:

- each $l_{i} \rightarrow r_{i}$-redex that occurs at a position $p$ in an $l_{j} \rightarrow r_{j}$-redex with $i \neq j$ occurs such that there exists a position $q \leq p$ with $q \in \mathcal{P}$ os $\left(l_{j}\right)$ and $\operatorname{root}\left(\left.l_{j}\right|_{p}\right)$ a meta-variable,

- likewise for $p \neq \epsilon$ and $i=j$.

2. $R$ is orthogonal if it is left-linear and non-overlapping.

3. An iCRS is orthogonal if its set of rewrite rules is orthogonal.

As shown in [10], orthogonality suffices for the definition of well-defined descendant and residual relations, i.e. the relations that describe respectively what "happens to" positions and redexes across reductions.

Notation 2.15. Let $s$ and $t$ be terms such that $s \rightarrow t$. Assume that $P \subseteq \mathcal{P}$ os $(s)$ and that $\mathcal{U}$ is a set of redexes in $s$. We denote descendants of $P$ across $s \rightarrow t$, respectively residuals of $\mathcal{U}$ across $s \rightarrow t$, by $P /(s \rightarrow t)$ and $\mathcal{U} /(s \rightarrow t)$. If $P=\{p\}$ and $\mathcal{U}=\{u\}$ we also write $p /(s \rightarrow t)$ and $u /(s \rightarrow t)$.

\section{Developments}

The results in this section apply to orthogonal iCRSs. Orthogonality is required, as descendants and residuals are only defined in the orthogonal case. 
Definition 3.1. Let $\mathcal{U}$ be a set of redexes in a term s. A development of $\mathcal{U}$ is a strongly convergent reduction sequence such that each step contracts a residual of a redex in $\mathcal{U}$. A development $s \rightarrow t$ is complete if $\mathcal{U} /(s \rightarrow t)=\emptyset$.

Notation 3.2. If $\mathcal{U}$ is a set of redexes in term $s$ and there is some development of $\mathcal{U}$ that results in term $t$, we write $s \Rightarrow t$, where the arrow is adorned with $\mathcal{U}$ if needed. Observe that there may exist $t^{\prime} \neq t$ with $s \Rightarrow t^{\prime}$, as the development $s \Rightarrow t$ need not to be complete.

The following is the main result of [10]:

Theorem 3.3. Let $\mathcal{U}$ be a set of redexes in a term s. If $\mathcal{U}$ has a complete development then all complete developments of $\mathcal{U}$ end in the same term.

Lemma 3.4. If $\mathcal{U}$ has a complete development and if $s \rightarrow t$ is a development of $\mathcal{U}$ (not necessarily complete), then $\mathcal{U} /(s \rightarrow t)$ has a complete development.

Proof. Immediate by inspection of the proof of Theorem 5.12(1) in [10].

Lemma 3.5. Let $\mathcal{U}$ be a set of redexes in a term $s$, let $\mathcal{U}$ have a complete development, and let $u$ be a redex in $s . T h e n \mathcal{U} \cup\{u\}$ has a complete development.

Proof (Sketch). By the finite chain condition on meta-terms and the variable convention, residuals of $u$ can only be nested in "finite chains" across a complete development of $\mathcal{U}$. One can coinductively perform complete developments of these finite chains in a top-down manner, yielding a complete development of $\mathcal{U} \cup\{u\}$.

Corollary 3.6. Let $\mathcal{U}$ be a set of redexes in a term $s$ which has a complete development $s \rightarrow t$ and let $v$ be a redex of $s$. The following diagram commutes (where all developments are complete):

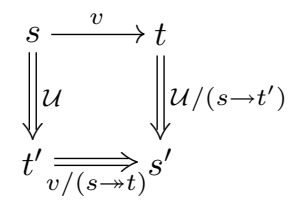

Proof. By Lemmas 3.4 and 3.5, Theorem 3.3 and the fact that $(\mathcal{U} \cup\{v\}) /(s \rightarrow$ $\left.t^{\prime}\right)=\mathcal{U} /\left(s \rightarrow t^{\prime}\right)$, respectively $(\mathcal{U} \cup\{v\}) /(s \rightarrow t)=v /(s \rightarrow t)$.

\section{Hypercollapsingness and Essentiality}

From this section onwards we consider only fully-extended, orthogonal iCRSs where each rewrite rule has a finite right-hand side. Finiteness of the right-hand sides is essentially used to show that Definition 4.11 is well-defined ${ }^{3}$.

In this section, we treat a special kind of troublesome term and reduction:

\footnotetext{
${ }^{3}$ The restriction to finite right-hand sides is crucial to the technique of considering essentiality that we employ in our proofs. We conjecture that it is possible to lift the restriction.
} 
Definition 4.1. A hypercollapsing reduction is a sequence of terms $\left(s_{i}\right)_{i<\omega}$ such that $s_{i} \rightarrow s_{i+1}$ for all $i<\omega$ and such that an infinite number of these steps are root-collapsing.

Thus, a hypercollapsing reduction is a transfinite reduction sequence of length $\omega$ which is not convergent in any sense and from which the term $s_{\omega}$ is omitted.

Definition 4.2. A term $s$ is said to be hypercollapsing if, for all terms $t$ with $s \rightarrow t$, there exists a term $t^{\prime}$ with $t \rightarrow t^{\prime}$ such that $t^{\prime}$ has a collapsing redex at the root.

The objective of this section is to prove the following lemma:

Lemma 4.3. Let $s$ be a term. If there is a hypercollapsing reduction starting from $s$, then $s$ is hypercollapsing.

This result is key for results concerning confluence modulo in iTRSs and i $\lambda$ c. Alas, the existing proof methods [6] cannot be lifted to the general higherorder case: For iTRSs, the known proofs hinge on the Strip Lemma, and for $\mathrm{i} \lambda \mathrm{c}$ on head reductions, none of which generalise to iCRSs. Instead, we employ a measure on finite reduction sequences and proof technique as developed by Sekar and Ramakrishnan [15] and as extended to higher-order rewriting by Van Oostrom [16].

\subsection{Essential Reductions}

To define the measure on finite reduction sequences, we first need to define the notions of contribution and essentiality.

Definition 4.4. Let $s$ and $t$ be terms and $s \rightarrow t$ with an $l \rightarrow r$-redex contracted at position $p$. If $q \in \mathcal{P}$ os $(s)$ and $P \subseteq \mathcal{P}$ os $(t)$, then $q$ contributes to $P$, whenever:

- one or more positions of $q /(s \rightarrow t)$ are in $P$, or

- the position $q$ occurs in the redex pattern of the contracted redex and $p$ is a prefix of some positions in $P$.

Contribution is extended to finite reductions of positive length by transitive closure. If $s \rightarrow^{=} s$, then every position in $P$ contributes only to itself.

Observe that several distinct positions in $s$ can contribute to a single position in $t$. In the case the redex contracted in $s \rightarrow t$ occurs at position $p$, at least all positions in the redex pattern contribute to the position $p$ in $t$.

Definition 4.5. Let $s \rightarrow^{*} t$ and let $P \subseteq \mathcal{P}$ os $(t)$. A position in any term along $s \rightarrow^{*} t$ is essential for $P$ (usually the explicit mention of $P$ is suppressed) if it contributes to $P$. A set of positions is essential for $P$ if every position in the set is. A redex is essential for $P$ if its root position contributes to $P$. A rewrite step is essential for $P$ if its redex is. A finite reduction is essential for $P$ if all of its rewrite steps are. A redex is inessential if its root position does not contribute to P. A rewrite step is inessential if its redex is. 
Lemma 4.6. A rewrite step is either essential or inessential.

Proof. By the fact that all positions in a redex pattern contribute to a redex.

Definition 4.7. A prefix of a term $s$ is a finite set $P \subseteq \mathcal{P}$ os $(s)$ such that all prefixes of positions in $P$ are also in $P$.

Take heed that prefixes are finite.

Lemma 4.8. Let $s_{0} \rightarrow^{*} s_{n}$ and let $P$ be a prefix of $s_{n}$. The positions in $s_{0}$ that are essential for $P$ form a prefix of $s_{0}$.

Proof. By induction on $n$. If $n=0$, we are done, since the reduction is empty. If $n=n^{\prime}+1$, then $P$ consists of a (possibly empty) set of positions $P^{\prime}$ "created" by the right-hand side of the redex contracted in the step $s_{n}^{\prime} \rightarrow s_{n}$ and a (possibly empty) set of positions descending from positions $Q$ in $s_{n^{\prime}}$. The positions contributing to $P^{\prime}$ are exactly the positions that occur in the redex pattern of the redex contracted in $s_{n^{\prime}} \rightarrow s_{n}$, and $Q$ consists of any position above or parallel to the redex, and of positions in arguments of the redex. The union of all these positions clearly constitutes a prefix of $s_{n^{\prime}}$. The induction hypothesis now furnishes the result.

By the above lemma, we may consider $s_{0} \rightarrow^{*} s_{n}$ as a sequence of $n$ prefixes such that each step either is inside the prefix of its term (and is hence essential), or is below the prefix (and is hence inessential).

Lemma 4.9. Let $s_{0} \rightarrow^{*} s_{n}$ and let $P$ be a prefix of $s_{n}$. There exists a reduction $s_{0} \rightarrow^{*} s^{\prime} \rightarrow s_{n}$ where $s_{1} \rightarrow^{*} s^{\prime}$ consists of steps essential for $P$ and $s^{\prime} \rightarrow s_{n}$ consists of steps inessential for $P$ (hence the prefix $P$ exists in $s^{\prime}$ ).

Proof. It suffices to show that if $t_{i} \Rightarrow t_{i}^{\prime} \rightarrow t_{i+1}$ where $t_{i} \Rightarrow t_{i}^{\prime}$ consists of a complete development of some set of redexes that contracts only inessential steps and $t_{i}^{\prime} \rightarrow t_{i+1}$ is an essential step, then $t_{i} \rightarrow t_{i}^{\prime \prime} \Rightarrow t_{i+1}$ for some term $t_{i}^{\prime \prime}$. Observe that since $t_{i} \Rightarrow t_{i}^{\prime}$ is inessential, the prefix of $t_{i}$ will not be touched by any step in $t_{i} \Rightarrow t_{i}^{\prime}$. Hence, the redex contracted in $t_{i}^{\prime} \rightarrow t_{i+1}$ is the unique residual of an essential redex in $t_{i}$. By Corollary 3.6 there now exists a term $t_{i}^{\prime \prime}$ such that $t_{i} \rightarrow t_{i}^{\prime \prime} \Rightarrow t_{i+1}$.

Notation 4.10. With the notation of the above lemma, we write $s_{0} \rightarrow^{*} s^{\prime}$ as $D_{e}$ (' $e$ ' for 'essential') and $s^{\prime} \rightarrow s_{n}$ as $D_{\bar{e}}$ (' $\bar{e}$ ' for 'inessential').

Definition 4.11. Let $D: s_{0} \Rightarrow^{\mathcal{U}_{1}} s_{1} \Rightarrow^{\mathcal{U}_{2}} \ldots \Rightarrow^{\mathcal{U}_{n}} s_{n}$ be a reduction consisting of a finite number of developments of finite sets of redexes (with finite righthand sides). The measure, $\mu(D)$ of $D$ is the $n$-tuple $\left(l_{n}, \ldots, l_{1}\right)$-note the reverse ordering! - where $l_{i}$ is the maximal length of a development of $\mathcal{U}_{i}$ that contracts only essential steps. Tuples are compared first by their length and then by their successive elements (in the natural order). This yields a well-founded order $\prec$. 
Note that the Finite Developments Theorem for ordinary CRSs applies: All developments of a finite set of redexes (with finite right-hand sides) are finite and end in the same term, and all maximal developments of such sets are complete [17]. Hence, each $l_{i}$ in the definition is well-defined.

Remark 4.12. Let $s_{0} \Rightarrow s_{1} \Rightarrow \cdots \Rightarrow s_{n}=D_{1} ; D_{2} ; \cdots ; D_{n}$ be a finite reduction consisting of developments of finite sets contracting only redexes essential to some prefix $P$ of $s_{n}$. In the remainder of this section we will consider a special kind of projection of such a reduction across a step $u: s_{0} \rightarrow t_{0}$ contracting a redex $u$. By applying the Finite Developments Theorem for finite CRSs to each single rewrite step in each $D_{i}$, we can erect the following diagram, in which each development is finite (but not necessarily complete):

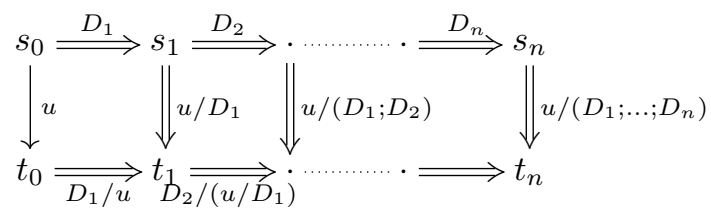

If $u$ is inessential, then it is outside the sequence of prefixes in $s_{0} \Rightarrow^{*} s_{n}$ contributing to $P$. Therefore, all bottommost steps in the above diagram are essential, and $P$ is a prefix of $t_{n}$.

If $u$ is essential and some residual of the redex of $u$ occurs in $D_{i}$, then some of the steps in the development $D_{i} /\left(u / D_{1} ; \ldots ; D_{i-1}\right)$ may be inessential, since redexes may have been duplicated by $u$ and since not all copies need to be essential. If this is the case, Lemma 4.9 ensures that we can rearrange $t_{0} \Rightarrow^{*} t_{n}$ an essential initial part $t_{0} \rightarrow^{*} q$ and an inessential final part $q \rightarrow t_{n}$ (such that $P$ is a prefix of the term $q$ ). We can thus "strip away" all inessential steps in the original projection to obtain an "emaciated" projection $t_{0} \rightarrow^{*} q$; observe that, in this case, we do not necessarily have $s_{n} \rightarrow q$.

The above remark ensures that the following definition is meaningful:

Definition 4.13. Let $s_{0} \Rightarrow s_{1} \Rightarrow \cdots \Rightarrow s_{n}=D_{1} ; D_{2} ; \cdots ; D_{n}$ be a finite reduction consisting of developments of finite sets contracting only redexes essential to some prefix $P$ for $s_{n}$. Let $s_{0} \rightarrow t_{0}$ contract a redex $u$. The emaciated projection of $D_{1} ; \cdots D_{n}$ across $u$, with respect to $P$, written $D / / u$ is the usual projection where inessential steps have been stripped out as in Remark 4.12.

Proposition 4.14. Let $D: s_{0} \Rightarrow^{*} s_{n}$, let $P$ be a prefix of $s_{n}$ and let $s_{0} \rightarrow t_{0}$ contract a redex $u$. Then, in the emaciated projection $D / / u: t_{0} \Rightarrow^{*} q$, the term $q$ contains $P$ as a prefix and $D / / u$ contains only essential steps for $P$.

Proof. This is the content of Remark 4.12 .

We want to relate the measure of the emaciated projections to the original reductions. The following two lemmas ensure that this can be done: 
Lemma 4.15. If $D$ factors as $D_{e} ; D_{\bar{e}}$ (according to Lemma 4.9), then $\mu\left(D_{e}\right) \preceq$ $\mu(D)$.

Proof. Inessential steps are not counted by $\mu$. In the proof of Lemma 4.9, the number of essential steps is constant under the permutation, whence the result.

Lemma 4.16. If $D: s_{0} \Rightarrow^{*} s_{n}, \mu(D)=\left(l_{n}, \ldots, l_{1}\right)$, and $s_{0} \rightarrow t_{0}$ contracts an essential redex $u$, then $\mu(D / / u) \prec \mu(D)$.

Proof. If $u$ is essential, then a residual of $u$ that is essential is contracted in one of the steps $s_{i} \Rightarrow{ }_{i}^{\mathcal{U}} s_{i+1}$. Assume that $i$ is the largest index of a set $\mathcal{U}_{i}$ such that $\mathcal{U}_{i}$ contains a residual $u^{\prime}$ of $u$ that is essential. If $u^{\prime}$ is the sole redex in $\mathcal{U}_{i}$, then the $i$ th component of $D / u$ becomes empty, and $\mu(D / u)$ will have length at least one less than $\mu(D)$. By Lemma 4.15 we have $\mu(D / / u) \preceq \mu(D / u)$. Hence, we obtain $\mu(D / / u) \prec \mu(D)$.

If $u^{\prime}$ is not the sole redex in $\mathcal{U}_{i}$, then write $\mu(D / / u)=\left(l_{n}^{\prime}, \ldots, l_{1}^{\prime}\right)$ and notice that $u$ may duplicate redexes from $\mathcal{U}_{1}, \ldots, \mathcal{U}_{i-1}$ Hence, increase the maximal length of their essential developments, i.e. we may have $l_{j}^{\prime}>l_{j}$ for $j<i$. However, the maximal length of a partial development of $\mathcal{U}_{i}$ that contracts only essential steps is now at least one less. Hence, $l_{i}^{\prime}<l_{i}$, and for all $l_{j}$ with $j>i$ we have $l_{j}^{\prime}=l_{j}$. Thus, $\mu(D / / u) \prec \mu(D)$.

Lemma 4.17. Suppose $D: s_{0} \rightarrow^{*} s_{n}$ is a reduction to a root-collapsing term and suppose $s_{0} \rightarrow t_{0}$ contracting a redex $u$ is not root-collapsing. Then, $t_{0}$ reduces to a root-collapsing term in a finite number of steps.

Proof. We may assume that $s_{0} \rightarrow^{*} s_{n}$ does not contain any root-collapsing steps (minimality). This implies that the collapsing redex at the root of $s_{0}$ is either (1) created along the reduction, or (2) that it was already at the root in $s_{0}$ (which implies $n=0$ ). If it was already in $s_{0}$, but at some other position than the root, then a root-collapsing step must occur in $s_{0} \rightarrow^{*} s_{n}$ (otherwise the root-collapsing redex can never be at the root), which is impossible by minimality.

Let $P$ be the set of positions in the redex pattern of the root-collapsing redex of $s_{n}$, and consider the emaciated projection $D / / u$. Since $u$ is not rootcollapsing, we have in the case of (1) that the final term $q$ in the emaciated projection must also be root-collapsing. In the case of (2) this is also holds, as the redex contracted in $s_{0} \rightarrow t_{0}$ must differ from the root-collapsing one in $s_{0}$, by the assumption on $s_{0} \rightarrow t_{0}$.

Lemma 4.18. If $s \rightarrow t$ contains no root-collapsing steps and $s$ reduces to a collapsing redex, then so does $t$.

Proof. If $s \rightarrow t$ is finite, the result follows by repeated application of Lemma 4.17 .

If $s \rightarrow t$ is infinite, we may by compression assume that it has length $\omega$ and by strong convergence that $s \rightarrow^{*} s_{n}$ by a finite reduction $D$ where $s_{n}$ is a collapsing redex. Let $P$ be the set of positions in the redex pattern of the root-collapsing 
redex in $s_{n}$. By Lemma, 4.8, the set of positions in $s$ that contribute to $P$ form a prefix $Q$ of $s$ and by Lemma 4.9 the reduction $s \rightarrow^{*} s_{n}$ consists solely of essential steps. We write $s \Rightarrow s_{1} \Rightarrow \cdots \Rightarrow s_{n}$, and $\mu(D)=\left(l_{n}, \ldots, l_{1}\right)$.

Since $s \rightarrow t$ is infinite, it consists of a first step $s \rightarrow t_{1}$ contracting a redex $u$ and an infinite reduction $t_{1} \rightarrow t$. Taking the emaciated projection of $s \Rightarrow s_{1} \Rightarrow$ $\cdots \Rightarrow s_{n}$ over $u$ yields a reduction $D / / u=t_{1} \rightarrow^{*} s_{n}^{\prime}$ to a collapsing redex. If $u$ is inessential, then $\mu(D / / v) \preceq \mu(D)$. Otherwise, by Lemma 4.16, $\mu(D / / v) \prec \mu(D)$.

If, from some $t_{i}$ in $s \rightarrow t$ onwards, no step is essential, then all steps are outside the prefix $Q_{i}$ of $t_{i}$ that contributes to $P$, hence the final term $t$ contains a prefix that reduces to a collapsing redex in "the same way" as $t_{i}$ does. Assume, for contradiction, that there are an infinite number of essential steps in $s \rightarrow t$. Then, Lemma 4.16 furnishes that the measure of the emaciated projected sequence decreases strictly in each of these steps, contradicting well-foundedness of $\prec$.

\subsection{Hypercollapsing reductions imply hypercollapsingness}

The following is the iCRS analogue of Lemma 12.8.4 in [6] for iTRSs and strengthening for $\mathrm{i} \lambda \mathrm{c}$ :

Lemma 4.19. Let $s_{0}$ be a term. If there exists a hypercollapsing reduction starting from $s_{0}$, and a rewrite step $s_{0} \rightarrow t_{0}$, then there is a hypercollapsing reduction starting from $t_{0}$.

Proof (Sketch). By definition of hypercollapsing reductions we may write:

$$
s_{0} \rightarrow^{*} s_{0}^{\prime} \rightarrow s_{1} \rightarrow^{*} s_{1}^{\prime} \rightarrow s_{2} \rightarrow^{*} \cdots,
$$

with $s_{i} \rightarrow s_{i+1}$ a root-collapsing step and no root-collapsing steps in $s_{i} \rightarrow^{*} s_{i}^{\prime}$, for all $i \in \mathbb{N}$. By repeated application of Corollary 3.6 we can erect the following diagram, where $u$ takes on the rôle of the set $\mathcal{U}$ when the corollary is first applied:

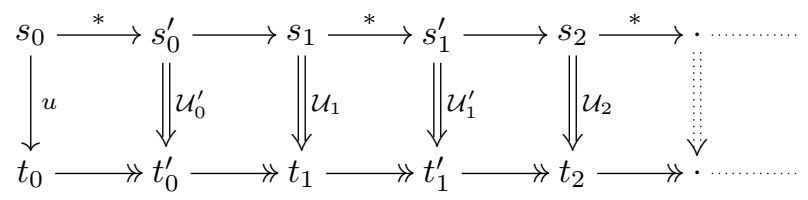

We write $S_{i}$ for $s_{i} \rightarrow s_{i}^{\prime} \rightarrow s_{i+1} \rightarrow \cdots$ and $T_{i}$ for $t_{i} \rightarrow t_{i}^{\prime} \rightarrow t_{i+1} \rightarrow \cdots$. Note that $T_{i}$ may have length greater than $\omega$.

If it holds for each $i \in \mathbb{N}$ that a root-collapsing step occurs in $T_{i}$, then an infinite number of root-collapsing steps occurs in $T_{0}$. We show this first. Afterwards, we extract a hypercollapsing reduction from $T_{0}$ employing this property.

To show the property we distinguish two cases: either (1) a root-collapsing step occurs in $S_{i}$ that does not contract a residual of $u$, or (2) all root-collapsing steps contract residuals of $u$. Careful case analysis shows that in both cases, $T_{i}$ will contain a root-collapsing step.

To show that a hypercollapsing reduction starting from $t_{0}$ exists, one repeatedly applies the case distinction above to $T_{i}$ for successively larger $i \in \mathbb{N}$. 
We can now prove Lemma 4.3:

Proof (Lemma 4.3). Let $s \rightarrow t$ and assume by compression that this reduction has length at most $\omega$. By strong convergence, we can write $s \rightarrow^{*} t^{\prime} \rightarrow t$ such that all root-reductions occur in $s \rightarrow^{*} t^{\prime}$. By repeated application of Lemma 4.19, there is a hypercollapsing reduction starting from $t^{\prime}$, in particular, $t^{\prime}$ reduces to a collapsing redex. Since $t^{\prime} \rightarrow t$ contains no steps at the root, Lemma 4.18 yields that $t$ reduces to a collapsing redex, proving that $s$ is hypercollapsing.

\section{Confluence Modulo}

We use the notion of a tiling diagram from [6]:

Definition 5.1. A tiling diagram of two strongly convergent reductions $S$ : $s_{0,0} \rightarrow^{\alpha} s_{\alpha, 0}$ and $T: s_{0,0} \rightarrow^{\beta} s_{0, \beta}$ is a rectangular arrangement of strongly convergent reductions:

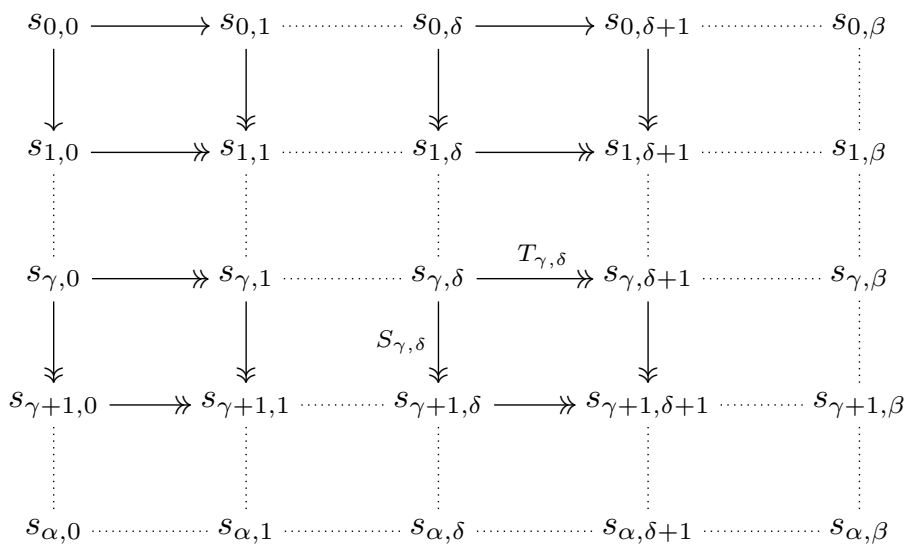

such that (1) each reduction $S_{\gamma, \delta}: s_{\gamma, \delta} \rightarrow s_{\gamma, \delta+1}$ is a complete development of a set of redexes of $s_{\gamma, \delta}$, and conversely with $T_{\gamma, \delta}: s_{\gamma, \delta} \rightarrow s_{\gamma+1, \delta}$, (2) the topmost horizontal reduction is $T$ and the leftmost vertical reduction is $S$, and (3) for each $\gamma, \delta$, the set of redexes developed in $T_{\gamma, \delta}$ is the set of residuals of the redex contracted in $s_{0, \delta} \rightarrow s_{0, \delta+1}$ across the (strongly convergent) reduction $S_{[0, \gamma], \delta}: s_{0, \delta} \rightarrow s_{1, \delta} \rightarrow \cdots s_{\gamma, \delta}$ (symmetrically for $S_{\gamma, \delta}$ ).

The below is part of Thm. 12.6.5 in [6]:

Theorem 5.2. Let $S$ and $T$ be strongly convergent reductions starting from the same term. The following are equivalent:

1. The tiling diagram of $S$ and $T$ can be completed, i.e. $S / T$ and $T / S$ are strongly convergent and have the same limit.

2. $S / T$ is strongly convergent.

3. $T / S$ is strongly convergent. 
Proof. The proof in [6] is independent of the details of rewriting.

Notation 5.3. By $s \rightarrow^{\text {out }} t$ we denote a rewrite step that does not occur inside any hypercollapsing subterm of $s$.

We now prove the analogue of Lemma 12.8.14 in [6]:

Lemma 5.4. If $S: s \rightarrow{ }^{\text {out }} t_{0}$ and $T: s \rightarrow{ }^{\text {out }} t_{1}$, then for some term $q$, we have $t_{0} \rightarrow q$ and $t_{1} \rightarrow q$.

Proof. Let $s \rightarrow^{\text {out }} t_{0}$ have length $\alpha$ and $s \rightarrow^{\text {out }} t_{1}$ have length $\beta$, respectively. Assume without loss of generality that $\alpha \leq \beta$, and proceed by induction on $\beta$ (the "outer" induction). Each case in this induction is in turn performed by induction on $\alpha$ (the "inner" induction). Induction ensures that it suffices to give a proof for $\alpha=\beta=1$, for $\alpha=1$ and $\beta=\omega$ (by Compression), and for $\alpha=\beta=\omega$.

- The case $\alpha=\beta=1$ is covered by Lemma 3.5.

- For the case $\alpha=1$ and $\beta=\omega$, Theorem 5.2 ensures that we need only prove that $\mathrm{S} / \mathrm{T}$ is strongly convergent, indeed since $S$ contracts a single redex $u$ we need only prove that the set $u / T$ has a strongly convergent complete development. Assume the contrary. Observe that only residuals of $u$ are contracted in any development of $u / T$ and that the employed rewrite rule is collapsing (otherwise any development $u / T$ is strongly convergent). As contracting residuals of $u$ cannot create further nestings of the residuals that are left, there exists a subterm of $t_{1}$ with a hypercollapsing reduction starting from it (obtained by a development of $u / T$ ), say at position $p$. In fact, there must exist an infinite chain of nested residuals of $u$ in the subterm at $p$. By strong convergence and limit length of $T$, we can write $T=T^{\prime \prime} ; T^{\prime}$ where $T^{\prime}: t \rightarrow^{\text {out }} t_{1}$ is a non-empty final segment of $T$ that performs no steps at prefix positions of $p$. Note that $T^{\prime \prime}$ is finite, by strong convergence. Thus, we have $\left.\left.t\right|_{p} \rightarrow{ }^{\text {out }} t_{1}\right|_{p}$. Since there is a hypercollapsing reduction starting from $\left.t_{1}\right|_{p}$, there is also a hypercollapsing reduction starting from $\left.t\right|_{p}$ interleaving the steps from $\left.\left.t\right|_{p} \rightarrow^{\text {out }} t_{1}\right|_{p}$ and the hypercollapsing reduction starting from $\left.t_{1}\right|_{p}$. But then by Lemma 4.3 we have that $\left.t\right|_{p}$ is hypercollapsing, which implies that $\left.\left.t\right|_{p} \rightarrow{ }^{\text {out }} t_{1}\right|_{p}$ is empty and that $\left.t\right|_{p}=\left.t_{1}\right|_{p}$. Thus, $\left.t\right|_{p}$ contains a set of descendants of $u$ having no complete development (giving rise to the hypercollapsing reduction from $\left.t\right|_{p}$ ), whence $u / T^{\prime \prime}$ has no complete development. Since $T^{\prime \prime}$ has length less than $\omega$, this contradicts the (outer) induction hypothesis.

- When $\alpha=\beta=\omega$, the argument from the proof of Lemma 12.8.14 in [6] can be copied verbatim, as it is independent of the details of rewriting.

Define $s \sim_{h c} t$ if and only if $t$ can be obtained from $s$ by replacing a number of hypercollapsing subterms of $s$ by other hypercollapsing terms. By orthogonality, $\sim_{h c}$ is an equivalence relation, which is closed under substitution of terms for free variables.

Lemma 5.5. If $s \rightarrow t$ and $s \sim_{h c} s^{\prime}$, then $s^{\prime} \rightarrow$ out $t^{\prime}$ and $t \sim_{h c} t^{\prime}$. 
Proof. Let $s \rightarrow^{\alpha} t$ and $s \sim_{h c} s^{\prime}$. We prove the result by transfinite induction.

- If $\alpha=0$, then the result is immediate, as an empty reduction sequence is by definition one that only contracts redexes outside hypercollapsing subterms.

- If $\alpha=\beta+1$, then assume $s \rightarrow^{\alpha} t=s \rightarrow^{\beta} q \rightarrow t$. By induction hypothesis we have that there exist $q^{\prime}$ such that $s^{\prime} \rightarrow^{\text {out }} q^{\prime}$ and $q \sim_{h c} q^{\prime}$. There are now two possibilities for $q \rightarrow t$, depending on the contracted redex occurring either outside or inside a hypercollapsing subterm:

- If the redex occurs outside a hypercollapsing subterm, then we have by $q \sim_{h c} q^{\prime}$ and orthogonality that a redex employing the same rewrite rule occurs at the same position in $q^{\prime}$ and that this redex occurs outside a hypercollapsing subterm. By definition of $\sim_{h c}$, contracting the redex in $q^{\prime}$ yields a term $t^{\prime}$ by a reduction outside a hypercollapsing subterm. That $t \sim_{h c} t^{\prime}$ follows by the fact that the same rewrite rule is employed in $q \rightarrow t$ and $q^{\prime} \rightarrow t^{\prime}$ and the fact that $q \sim_{h c} q^{\prime}$ : Clearly, $t$ and $t^{\prime}$ are identical at all positions $p$ that descend from positions not in hypercollapsing subterms of $q$ or $q^{\prime}$. If $p^{\prime}$ is the position of a maximal hypercollapsing subterm of $q$, it is also the position of a maximal hypercollapsing subterm of $q^{\prime}$ and vice versa, and $p^{\prime}$ descends to identical positions in $t$, respectively $t^{\prime}$. Any descendant of the subterm at $p^{\prime}$ will be a (not necessarily maximal) hypercollapsing subterm, and the result then follows by $q \sim_{h c} q^{\prime}$ and its closure under substitution.

- If the redex occurs inside a hypercollapsing subterm, then we have $t \sim_{h c}$ $q$. Hence, by transitivity of $\sim_{h c}$ we have $t \sim_{h c} q^{\prime}$ and we can define $t^{\prime}=q^{\prime}$.

- If $\alpha=\gamma$, with $\gamma$ a limit ordinal, then the result is immediate by strong convergence and the induction hypothesis.

Definition 5.6. An iCRS is said to be confluent modulo an equivalence relation $\sim$ if $s \sim t, s \rightarrow s^{\prime}$, and $t \rightarrow t^{\prime}$ imply existence of terms $s^{\prime \prime}$ and $t^{\prime \prime}$ such that $s^{\prime} \rightarrow s^{\prime \prime}, t^{\prime} \rightarrow t^{\prime \prime}$ and $s^{\prime \prime} \sim t^{\prime \prime}$.

Theorem 5.7. Fully-extended, orthogonal iCRSs with finite right-hand sides are confluent modulo $\sim_{h c}$.

Proof. Let $s \sim_{h c} t$, and assume that $s \rightarrow s^{\prime}$ and $t \rightarrow t^{\prime}$. Consider the following diagram:

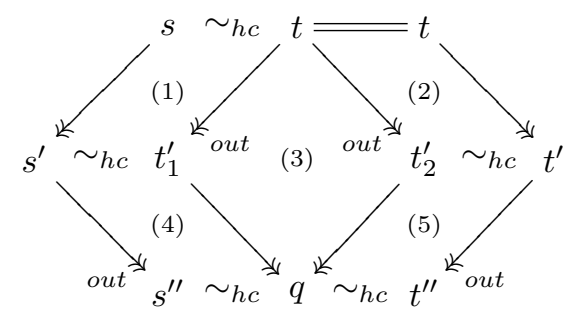

Prisms (1) and (2) follow by Lemma 5.5. Square (3) follows by Lemma 5.4. The diagram is completed by noting that (4) and (5) follow by Lemma 5.5 The result now follows by transitivity of $\sim_{h c}$. 


\section{References}

1. Hanus, M.: A unified computation model for functional and logic programming. In: Proceedings of the 24th Annual SIGPLAN-SIGACT Symposium on Principles of Programming Languages (POPL '97), ACM Press (1997) 80-93

2. Albert, E., Hanus, M., Huch, F., Oliver, J., Vidal, G.: An operational semantics for declarative multi-paradigm languages. In: Proceedings of the 11th International Workshop on Functional and (Constraint) Logic Programming (WFLP '02), Università degli Studi di Udine (2002) 7-20

3. Fernández, A.J., Hortalá-Gonzales, T., Sáenz-Pérez, F.: Solving combinatorial problems with a constraint functional logic language. In: Practical Aspects of Declarative Languages (PADL '03). Volume 2562 of LNCS., Springer-Verlag (2003) 320-338

4. Dershowitz, N., Kaplan, S., Plaisted, D.A.: Rewrite, rewrite, rewrite, rewrite, rewrite, .... Theoretical Computer Science 83 (1991) 71-96

5. Kennaway, R., Klop, J.W., Sleep, R., de Vries, F.J.: Transfinite reductions in orthogonal term rewriting systems. Information and Computation 119 (1995) 1838

6. Terese: Term Rewriting Systems. Cambridge University Press (2003)

7. Kennaway, J.R., Klop, J.W., Sleep, M., de Vries, F.J.: Infinitary lambda calculus. Theoretical Computer Science 175 (1997) 93-125

8. Marchiori, M.: Logic programs as term rewriting systems. In: Proceedings of the 4th International Conference on Algebraic and Logic Programming. Volume 850 of LNCS., Springer-Verlag (1994) 223-241

9. van Raamsdonk, F.: Translating logic programs into conditional rewriting systems. In: Proceedings of the 14th International Conference on Logic Programming (ICLP '97), MIT Press (1997) 168-182

10. Ketema, J., Simonsen, J.G.: Infinitary combinatory reduction systems. In Giesl, J., ed.: Proceedings of the 16th International Conference on Rewriting Techniques and Applications (RTA '05). Volume 3467 of LNCS., Springer-Verlag (2005) 438-452

11. Klop, J.W., van Oostrom, V., van Raamsdonk, F.: Combinatory reduction systems: introduction and survey. Theoretical Computer Science 121 (1993) 279-308

12. Arnold, A., Nivat, M.: The metric space of infinite trees. Algebraic and topological properties. Fundamenta Informaticae 3 (1980) 445-476

13. Hanus, M., Prehofer, C.: Higher-order narrowing with definitional trees. In Ganzinger, H., ed.: Proc. of the 7th Int. Conf. on Rewriting Techniques and Applications (RTA'96). Volume 1103 of LNCS., Springer-Verlag (1996) 138-152

14. van Oostrom, V.: Higher-order families. In Ganzinger, H., ed.: Proc. of the 7th Int. Conf. on Rewriting Techniques and Applications (RTA '96). Volume 1103 of LNCS., Springer-Verlag (1996) 392-407

15. Sekar, R.C., Ramakrishnan, I.V.: Programming in equational logic: beyond strong sequentiality. Information and Computation 104 (1993) 78-109

16. van Oostrom, V.: Normalisation in weakly orthogonal rewriting. In: Proceedings of the 10th International Conference on Rewriting Techniques and Applications (RTA '99). Volume 1631 of LNCS., Springer-Verlag (1999) 60-74

17. Klop, J.W.: Combinatory Reduction Systems. PhD thesis, Rijksuniversiteit Utrecht (1980)

18. Barendregt, H.P.: The Lambda Calculus: Its Syntax and Semantics. Revised edn. Elsevier Science (1984) 